\title{
The WRKY Superfamily of Rice Transcription Factors
}

\author{
Ji-Young Jang, Chang Hyun Choi and Duk-Ju Hwang* \\ National Academy of Agricultural Sciences, Rural Development Administration, Suwon 441-707, Korea
}

(Received on January 12, 2010; Accepted on May 9, 2010)

\begin{abstract}
WRKY transcription factors are known to be involved in many different biological processes including plant response to biotic stress, abiotic stress, and plant development. WRKY proteins are extensively studied in Arabidopsis. Recently, reports on WRKY proteins are rapidly increasing in the other plant species, especially in rice. Therefore, this review will discuss the function of rice WRKY proteins reported so far.
\end{abstract}

Keywords : defense response, jasmonic acid, rice, salicylic acid, WRKY transcription factor

Plants are constantly under plethora of stresses and developmental changes. To adapt plant to new environments it has to go through massive transcriptional changes of the related genes. Transcription factors play major roles in the transcriptional changes of the related genes. WRKY proteins are involved in growth development such as embryogenesis (Lagace et al., 2004), trichome development (Johnson et al., 2002), senescence (Hinderhofer et al., 2001; Robatzek et al., 2001) and plant responses to various stresses such as biotic stress (Eulgem et al. 2007; Pandey et al., 2009), abiotic stress (Wu et al., 2009). Since the first cDNA encoding a WRKY protein, SPF1, was cloned from sweet potato (Ipomoea batatas) (Ishiguro et al., 1994), the number of WRKY genes have been reported in the Arabidopsis and other plant species. WRKY proteins are extensively studied in Arabidopsis. Recently the number of reports is rapidly accumulating on Orysa sativa WRKY (OsWRKY) transcription factors. Therefore, it will be useful to review reports on the function of $O s \mathrm{WRKY}$ members reported so far.

\section{The common characteristics of WRKY transcription factor}

The WRKY superfamily of plant transcription factors (TF) consists of 74 and 109 members in Arabidopsis and rice, respectively (Eulgem et al., 2007; Liu et al., 2007;

\footnotetext{
*Corresponding author.

Phone) +82-31-299-1742, FAX) +82-31-299-1722

E-mail)djhwang@korea.kr
}

Ramamoorthy et al., 2008; Ross et al., 2007; Wu et al., 2005). WRKY TFs contain at least one conserved DNAbinding region, called as the WRKY domain. The WRKY domain comprises the highly conserved WRKYGQK peptide sequence and a zinc finger motif $\left(\mathrm{CX}_{4-7} \mathrm{CX}_{22-23}\right.$ $\mathrm{HXH} / \mathrm{C}$ ). This domain is responsible for binding to the $\mathrm{W}$ box (C/TTGACT/C), although alternative binding sites have been identified (Cai et al., 2008; Ciolkowski et al., 2008; Sun et al., 2003; Van Verk et al., 2008). Variants of the WRKYGQK signature motif include WRKYGKK and WRKYGEK, and several atypical WRKY domains suggest the consensus $\mathrm{W}(\mathrm{R} / \mathrm{K})(\mathrm{K} / \mathrm{R}) \mathrm{Y}$ (Xie et al., 2005). WRKY proteins possess one or more WRKY domains. Based on the nature of the DNA binding domain and the number of WRKY domain, WRKY transcription factors are categorized into either three or four groups (Eulgem et al., 2000; Ross et al., 2007). WRKY proteins containing a single WRKY domain with $\mathrm{C}_{2}-\mathrm{H}_{2}$ pattern belong to group II; those containing two WRKY domains with $\mathrm{C}_{2}-\mathrm{H}_{2}$ pattern are group I; the others containing a WRKY domain with $\mathrm{C}_{2}$-HC pattern belong to group III. Two research groups proposed group IV especially for OsWRKY proteins that contain a WRKY domain but lack a complete zinc finger (Ross et al., 2007; Xie et al., 2005). The group II WRKY proteins are further subdivided into subgroups a-e based on the presence of short conserved structural motifs (Eulgem et al., 2000). For example, the group IId WRKY proteins have been shown to bind calmodulin through the defining non-classical calmodulin-binding domain (AcMBD) VSSFK(K/R)VISLL (Park et al., 2005). Zhang and Wang (2005) have also proposed a different set of subgroups based on phylogenetic relationships of $O s \mathrm{WRKY}$ proteins.

\section{Expression of OSWRKY transcription factors in res- ponse to various stimuli}

WRKY transcription factors are involved in many different biological processes such as response to pathogen attack, wounding, drought, cold, senescence, development, dormancy, solar ultraviolet B-radiation, metabolism, and hormones. Expression profiling analysis of plants treated with various stimuli revealed the occurrence of drastic changes of massive gene expression including various 
transcription factors. Expression profiles of genes are important for the investigation of the function of transcription factors. The number of Arabidopsis WRKY (AtWRKY) genes are induced by pathogen and biotic elicitors such as SA, JA, cell wall-derived elicitor (Chen et al., 2000). According to the previous report 15 OsWRKY genes were induced upon $M$. grisea infection (Ryu et al., 2006). In contrast, the other research group reported that $23 W R K Y$ genes were induced upon $M$. grisea infection (Ramamoorthy et al., 2009). Another group reported that OsWRKY4, OsWRKY23, OsWRKY24, OsWRKY38, OsWRKY53, OsWRKY61, OsWRKY63, OsWRKY100, OsWRKY101, OsWRKY108, OsWRKY110, and OsWRKY115 are induced 7-folds by $M$. grisea infection (Berri et al., 2009). This dissimilarity may come from different isolate of pathogen and different detection system such as RT-PCR and microarray used in their experimental systems. More recent expression profiling analysis of $O_{s} W R K Y s$ reveals that 42 member out of $98 W R K Y$ members from TIGR database are influenced in their expression level upon Xoo infection (Qiu et al., 2009). Twenty-seven of the 42 WRKY genes were up-regulated upon pathogen infection and the W-boxes are over-represented in the promoter region of upregulated genes, proposing that many OsWRKY genes are self-regulated by the WRKY family. Some WRKY genes are also induced by abiotic stresses. OsWRKY20, OsWRKY43, OsWRKY78, OsWRKY87, and OsWRKY100 are induced in above 7-folds by osmotic stress (Berri et al., 2009). OsWRKY50 and OsWRKY90 are induced by flooding; OsWRKY3, OsWRKY8, OsWRKY24, OsWRKY42, OsWRKY50, and $O s W R K Y 96$ are induced by cold and drought. Some $W R K Y$ genes are induced by hormones such as ABA and GA (Xie et al., 2005; Zhang et al., 2009). Induction of Os WRKY genes in response to various stimuli is proposing that $O s \mathrm{WRKY}$ proteins are involved in various biological processes as AtWRKY proteins.

\section{Roles of OsWRKY transcription factors in biotic stresses}

Plants have developed two layers of innate immunity system against pathogen attacks (Jones and Dangl, 2006). The first layer of innate immunity is triggered by pathogenassociated molecular patterns (PAMPs) such as flagellin, elongation factor Tu, and chitin, which is called PAMPtriggered immunity (PTI). The second layer of innate immunity is triggered by resistance $(\mathrm{R})$ proteins upon the recognition of corresponding effector proteins which is called effector-triggered immunity (ETI). Plant signaling molecules, such as salicylic acid (SA), jasmonic acid (JA) and ethylene play important roles in PTI and ETI signaling pathways (Chen et al., 2000; Dellagi et al., 2000; Du et al.,
2000; Eulgem et al., 2000; Kim et al., 2000; Asai et al., 2002; Ulker et al., 2004). During PTI and ETI plants go through massive transcriptional changes of defense related genes. Transcrption factors are responsible for transcriptional changes of defense related genes. It has been reported that the WRKY superfamily plays major roles in defense signaling pathways. Recent evidences are accumulating that common defense pathways in Arabidopsis are well conserved in rice (Chern et al., 2005). However, only few counter parts of genes known in Arabidopsis were reported in rice such as NPR1.

During the past decade, numerous reports have indicated that $W R K Y$ genes are involved in defense responses in rice. Over-expression of OsWRKY03 and OsWRKY71 exhibited enhanced resistance to Xanthomonas oryzae pv. oryzae $(X o o)$ and it functions upstream of $O s \mathrm{NH} 1$ (Liu et al., 2005; Liu et al., 2007). In the case of OsWRKY13, over-expressor lines showed enhanced resistance to Xoo and Magnaporthe grisea through activation of SA-dependent pathways and suppression of JA dependent pathways (Qiu et al., 2007). Expression profiling analysis of OsWRKY13 activated transgenic lines revealed that $O s W R K Y 10$ is up-regulated and OsWRKY14, OsWRKY24, OsWRKY42, OsWRKY45, OsWRKY51, OsWRKY68 are down-regulated. Down-regulated $O s W R K Y$ members seems to be negative regulator in OsWRKY13 mediated defense signaling. However, over-expression of OsWRKY45 also showed enhanced resistance to $M$. grisea and Xoo (Shimono et al., 2007; Tao et al., 2009); over-expression of OsWRKY71 enhances disease resistance to Xoo (Liu et al., 2007). OsWRKY24, OsWRKY51, and OsWRKY71 are known to be involved in the different signaling pathways (Xie et al., 2005; Xie et al., 2006). The detailed action mechanism between these OsWRKYs remains to be elucidated. OsWRKY31 overexpressor lines were resistant to $M$. grisea and showed constitutive expression of many defense related genes and auxin-responsive genes (Zhang et al., 2007). OsWRKY45 is initially identified as a benzothiadiazole (BTH) inducible OsWRKY (Shimono et al., 2007). Over-expression of OsWRKY45 isolated from japonica var. Nipponbare enhances rice blast resistance and its knock-down compromises BTH-inducible blast resistance. Interestingly overexpression of both OsWRKY45-1 from japonica var. Nipponbare and $O s W R K Y 45-2$ from indica var. Minghui 63 enhances rice blast resistance respectively, proposing that both $O s$ WRKY45-1 and OsWRKY45-2 are positive regulators in blast resistance (Tao et al., 2009). OsWRKY45-1 over-expressing plants show increased susceptibility to Xoo while OsWRKY45-2 over-expressing plants shows enhanced resistance to Xoo, proposing that a pair of allelic $W R K Y$ genes play opposite roles in rice-Xoo interactions. Their detailed mechanism of OsWRKY45-1 and OsWRKY45-2 
mediated response to $X o o$ need to be elucidated. Overexpression of OsWRKY53 also results in enhanced disease resistance to $M$. grisea and constitutive expression of defense related genes such as PBZ1, chitinase 1, PR5 etc. (Chujo et al., 2007). OsWRKY62 is initially characterized as a Xa21 binding protein (Peng et al., 2008). Overexpression of OsWRKY62 exhibit enhanced susceptibility to $X o O$ in wild type background and compromise disease resistance to Xoo in Xa21 background, proposing that OsWRKY62 is a negative regulator in basal resistance and Xa21-mediated defense to Xoo in rice. It remains to be elucidated how OsWRKY62 regulates the Xa21 mediated resistance in relation to interaction between $\mathrm{Xa} 21$ and OsWRKY62. Unlike other OsWRKYs, over-expression of OsWRKY89 confers enhanced resistance to rice blast fungus and white backed plant hopper as well as UV irradiation (Wang et al., 2007). Overall OsWRKY13, OsWRKY31, OsWRKY71 confers resistance to bacterial rice blight pathogen, Xoo. OsWRKY13, OsWRKY31, OsWRKY45, OsWRKY53, OsWRKY89 confers resistance to blast fungus, M. grisea. OsWRKY 13 and OsWRKY31 confer resistance to Xoo and Magnaporthe grisea. Interestingly, OsWRKY89 confers resistance to insects and rice blast fungus. Numerous WRKY proteins are involved in disease resistance in rice as in Arabidopsis.

\section{Roles of $O s W R K Y$ transcription factors in response to abiotic stresses and phytohormones}

WRKY transcription factors play a variety of developmental and physiological roles in plants. The WRKY transcription factors are involved in plant responses to abiotic stresses such freezing (Huang et al., 2002), wounding (Hara et al., 2000), oxidative stress (Rizhsky et al.,
2004), drought, salinity, cold, heat (Pnueli et al., 2002; Rizhsky et al., 2002; Seki et al., 2002) and ultraviolet B (Wang et al., 2007). A dozen rice WRKY genes are induced by heat shock, cold stress, high salinity, and polyethylene glycol (Qiu et al., 2004). OsWRKY11 was induced by heat and drought. Over-expression of OsWRKY11 under the control of HSP101 promoter showed enhanced heat and drought tolerance (Wu et al., 2009). OsWRKY31 overexpressor lines show enhanced disease resistance to $M$. grisea and also affect root growth and auxin response in transgenic rice plants (Zhang et al., 2008). Several rice $W R K Y$ genes are induced by phytohormone such as ABA and/or GA. The transient expression experiments demonstrated several rice $W R K Y$ genes are capable of regulating ABA-inducible $H V A 22$ promoter in positive (OsWRKY72 and $O s W R K Y 77$ ) or negative (OsWRKY24 and OsWRKY45) manner. OsWRKY11 and OsWRKY71 were induced by ABA, but they do not regulate the ABA-inducible HVA22 promoter in aleurone cells (Xie et al., 2005). The OsWRKY51 and OsWRKY71 mediate the cross-talk between GA and ABA signaling. These genes were ABA inducible but GA repressible in rice (Xie et al., 2006). OsWRKY24, OsWRKY51 and OsWRKY71 were negative regulator of GA signaling (Xie et al., 2005; Zhang et al., 2009).

\section{Conclusions}

WRKY transcription factors are induced by various stimuli including pathogen, SA, JA, ABA etc. The major reports of WRKY transcription factors are accumulated in relation to pathogen defense. But now many reports are accumulating that some WRKY transcription factors are involved in various physiological phenomena such as phytohormone response. Breakthrough technologies in this area will be

Table 1. List of rice $W R K Y$ genes that their functions are reported so far.

\begin{tabular}{|c|c|c|c|c|}
\hline Name & LOC & cDNA & Gene Function & References \\
\hline WRKY03 & Os03g55080 & AK069091 & Defense & Liu et al., 2005 \\
\hline WRKY11 & Os01g43650 & AK108745 & Heat and drought & Xiaolan et al., 2009 \\
\hline WRKY13 & Os01g54600 & AK067329 & Defense/SA/JA & Qiu et al., 2007, 2008 \\
\hline WRKY24 & Os01g61080 & AK107199 & GA/ABA & Zhang et al., 2009 \\
\hline WRKY31 & Os06g30860 & AK101653 & Defense/lateral root formation & Zhang et al., 2008 \\
\hline WRKY45 & Os05g25770 & AK066255 & Defense Defense/drought & Shimono et al., 2007, Qiu et al., 2009 \\
\hline WRKY51 & Os04g21950 & AK100954 & $\mathrm{ABA} / \mathrm{GA}$ & Xie et al., 2006 \\
\hline WRKY53 & Os05g27730 & AK121190 & Defense & Chujo et al., 2007 \\
\hline WRKY62 & Os09g25070 & AK067834 & Defense & Peng et al., 2008 \\
\hline WRKY71 & Os02g08440 & AB190817 & Defense $\mathrm{ABA} / \mathrm{GA}$ & Liu et al., 2007, Xie et al., 2006 \\
\hline WRKY72 & Os11g29870 & AK108860 & $\mathrm{ABA}$ & Xie et al., 2005 \\
\hline WRKY77 & Os01g40260 & AK108522 & $\mathrm{ABA}$ & Xie et al., 2005 \\
\hline WRKY89 & Os05g03900 & AY781112 & Defense/ultraviolet B & Wang et al., 2007 \\
\hline
\end{tabular}


high-throught screening methods to investigate target genes of WRKY transcription factors in post-genome era to dissect their detailed mechanism. Numerous reports suggest that OsWRKY genes can be used for crop plants with enhanced resistance to pathogen.

\section{Acknowledgments}

This work was supported in part by a grant CG3134-1 from 21st Century Frontier Crop Functional Genomics and grants from the National Academy of Agricultural Sciences, Rural Development Administration to Dr. Duk-Ju Hwang.

\section{References}

Asai, T., Tena, G, Plotnikova, J., Willmann, M. R., Chiu, W. L. and Gomez-Gomez, L. 2002. MAP kinase signaling cascade in Arabidopsis innate immunity. Nature 415:977-983.

Berri, S., Abbruscato, P., Faivre-Rampant, O., Brasileiro, A. C. M., Fumasoni, I., Satoh. K., Kikuchi, S., Mizzi, L., Morandini, P., Pe, M. E. and Piffanelli, P. 2009. Characterization of WRKY co-regulatory networks in rice and Arabidopsis. BMC Plant Biology 9:120-141

Cai, M., Qiu, D., Yuan, T., Ding, X., Li, H., Duan, L., Xu, C., Li, X. and Wang, S. 2008. Identification of novel pathogenresponsive cis-elements and their binding proteins in the promoter of OsWRKY13, a gene regulating rice disease resistance. Plant Cell Environ. 31:86-96.

Chen, C. and Chen, Z. 2000. Isolation and characterization of two pathogen- and salicylic acid-induced genes encoding WRKY DNA-binging proteins from tobacco. Plant Mol. Biol. 42:387396.

Chern, M., Fitzgerald, H. A., Canlas, P. E., Navarre, D. A. and Ronald, P. C. 2005. Overexpression of a rice NPR1 homolog leads to constitutive activation of defense response and hypersensitivity to light. Mol. Plant-Microbe Interact. 18:511-520.

Chujo, T., Takai, R., Akimoto-Tomiyama, C., Ando, S., Minami, E., Nagamura, Y., Kaku, H., Shibuya, N., Yasuda, M. and Nakashita, H. 2007. Involvement of the elicitor-induced gene OsWRKY53 in the expression of defense related genes in rice. Biochim. Biophys. Acta. 1769:497-505.

Ciolkowski, I., Wanke, D., Birkenbihl, R. and Somssich, I. 2008. Studies on DNA-binding selectivity of WRKY transcription factors lend structural clues into WRKY-domain function. Plant Mol. Biol. 68:81-92.

Dellagi, A., Helibronn, J., Avrova, A. O., Montesano, M., Palva, E. T. and Stewart, H. E. 2000. A potato gene encoding a WRKY-like transcription factor is induced in interactions with Erwinia carotovora subsp. Atroseptica and Phytophthora infestans and is coregulated with class I endochitinase expression. Mol. Plant-Microbe Interact. 13:1092-1101.

Du, L. and Chen, Z. 2000. Identification of genes encoding receptor-like protein kinases as possible targets of pathogen- and salicylic acid-induced WRKY DNA-binding proteins in Arabidopsis. Plant J. 24:837-847.

Eulgem, T., Rushton, P. J., Robatzek, S. and Somssich, I. E. 2000. The WRKY superfamily of plant transcription factors. Trends Plant Sci. 5:199-206.

Eulgem, T. and Somssich, I. E. 2007. Networks of WRKY transcription factors in defense signaling. Curr. Opin. Plant Biol. 10:366-371.

Hinderhofer, K. and Zentgraf, U. 2001. Identification of a transcription factor specifically expressed at the onset of leaf senescence. Planta 213:469-473.

Huang, T. and Duman, J. G. 2002. Cloning and characterization of a thermal hysteresis (antifreeze) protein with DNA-binding activity from winter bittersweet nightshade, Solanum dulcamara. Plant Mol. Biol. 48:339-350.

Hara, K., Yagi, M., Kusano, T. and Sano, H. 2000. Rapid systemic accumulation of transcripts encoding tobacco WRKY transcription factor upon wounding. Mol. Gen. Genet. 263:30-37.

Ishiguro, S. and Nakamura, K. 1994. Characterization of a cDNA encoding a novel DNA-binding protein, SPF1, that recognizes SP8 sequences in the 5' upstream regions coding for sporamin and beta-amylase from sweet potato. Mol. Gen. Genet. 244:563-571.

Johnson, C., Kolevski, B. and Smyth, D. 2002. TRANSPARENT TESTAGLABRA2, a trichome and seed coat development gene of Arabidopsis, encodes a WRKY transcription factor. Plant Cell 14:1359-1375.

Jones, J. D. and Dangl, J. L. 2006. The plant immune system. Nature 444:323-329.

Kim, C. Y., Lee, S. H., Park, H. C., Bae, C. G., Cheong, Y. H., Choi, Y. J., Han, C., Lee, S. Y., Lim, C. O. and Cho, M. J. 2000. Identification of rice blast fungal elicitor-responsive genes by differential display analysis. Mol. Plant-Microbe Interact. 13:470-474.

Lagace, M. and Matton, D. P. 2004. Characterization of a WRKY transcription factor expressed in late torpedo-stage embryos of Solanum chacoense. Planta 219:185-189.

Liu, X. Q., Bai, X. Q., Qian, Q., Wang, X. J., Chen, M. S. and Chu, C. C. 2005. OsWRKY03, a rice transcriptional activator that functions in defense signaling pathway upstream of OsNPR1. Cell Res. 15:593-603.

Liu, X. Q., Bai, X. Q., Wang, X. J. and Chu, C. C. 2007. OsWRKY71, a rice transcription factor, is involved in rice defense response. J. Plant Physiol. 164:969-979.

Pandey, S. P. and Somssich, I. E. 2009. The role of WRKY transcription factors in plant immunity. Plant Physiol. 150:164855

Park, C. Y., Lee, J. H., Yoo, J. H., Moon, B. C., Choi, M. S., Kang, Y. H. 2005. WRKY group IId transcription factors interact with calmodulin. FEBS Lett. 579:1545-1550.

Peng, Y., Bartley, L. E., Chen, X., Dardick, C., Chern, M., Ruan, R., Canlas, P. E. and Ronald, P. C. 2008. OsWRKY62 is a negative regulator of basal and Xa21-mediated defense against Xanthomonas oryzae pv. oryzae in rice. Mol. Plant 1:446-458.

Pnueli, L., Hallak-Herr, E., Rozenberg, M., Cohen, M., Goloubinoff, P., Kaplan, A. and Mittler, R. 2002. Molecular and bio- 
chemical mechanisms associated with dormancy and drought tolerance in the desert legume Retama raetam. Plant J. 31:319-330.

Qiu, Y., Jing, S., Fu, J., Li, L., Yu, D. 2004. Cloning and analysis of expression profile of 13 WRKY genes in rice. Chin. Sci. Bull. 49:2159-2168.

Qiu, D., Xiao, J., Ding, X., Xiong, M., Cai, M., Cao, Y., Li, X., $\mathrm{Xu}, \mathrm{C}$. and Wang, S. 2007. OsWRKY13 mediates rice disease resistance by regulating defense related genes in salicylateand jasmonate-dependent signaling. Mol. Plant-Microbe Interact. 20:492-499.

Qiu, D., Xiao, J., Xie, W., Liu, H., Li, X., Xiong, L. and Wang, S. 2008. Rice gene network inferred from expression profiling of plants overexpressing OsWRKY13, a positive regulator of disease resistance. Mol. Plant 1:538-551.

Qiu, Y. and Yu, D. 2009. Over-expression of the stress-induced OsWRKY45 enhances disease resistance and drought tolerance in Arabidopsis. Environ. Exp. Bot. 65:35-47.

Ramamoorthy, R., Jiang, S. Y., Kumar, N., Venkatesh, P. N. and Ramachandran, S. 2008. A comprehensive transcriptional profiling of the WRKY gene family in rice under various abiotic and phytohormone treatments. Plant Cell Physiol. 49:865879.

Rizhsky, L., Davletova, S., Liang, H. and Mittler, R. 2004. The zinc finger protein Zat12 is required for cytosolic ascorbate peroxidase 1 expression during oxidative stress in Arabidopsis. J. Biol. Chem. 279:11736-11743.

Rizhsky, L., Liang, H. and Mittler, R. 2002. The combined effect of drought stress and heat shock on gene expression in tobacco. Plant Physiol. 130:1143-1151.

Robatzek, S. and Somssich, I. E. 2001. A new member of the Arabidopsis WRKY transcription factor family, AtWRKY6, is associated with both senescence- and defence-related processes. Plant J. 28:123-133.

Ross, C. A., Liu, Y. and Shen, Q. J. 2007. The $W R K Y$ gene family in rice (Oryza sativa). J. Integr. Plant Biol. 49:827-842.

Ryu, H. S., Han, M., Lee, S. K., Cho, J. I., Ryoo, N., Heu, S., Lee, Y. H., Bhoo, S., Wang, G L. and Hahn, T. R. 2006. A comprehensive expression analysis of the $W R K Y$ gene superfamily in rice plants during defense response. Plant Cell Rep. 25:836847.

Seki, M., Narusaka, M., Ishida, J., Nanjo, T., Fujita, M., Oono, Y., Kamiya, A., Nakajima, M., Enju, A., Sakurai, T., Satou, M., Akiyama, K., Taji, T., Yamaguchi-shinozaki, K., Carninci, P., Kawai, J., Hayashizaki, Y. and Shinozaki, K. 2002. Monitoring the expression profiles of 7000 Arabidopsis genes under drought, cold and high-salinity stresses using a full-length cDNA microarray. Plant J. 31:279-292.

Shimono, M., Sugano, S., Nakayama, A., Jiang, C.-J., Ono, K., Toki, S. and Takatsuji, H. 2007. Rice WRKY45 plays a crucial role in benzothiadiazole inducible blast resistance. Plant Cell. 19:2064-2076.

Sun, C., Palmqvist, S., Olsson, H., Boren, M., Ahlandsberg, S. and Jansson, C. 2003. A novel WRKY transcription factor, SUSIBA2, participates in sugar signaling in barley by binding to the sugar responsive elements of the iso 1 promoter. Plant
Cell 15:2076-2092.

Tao, Z., Liu, H., Qiu, D., Zhou, Y., Li, X., Xu, C. and Wang, S. 2009. A pair of allelic $W R K Y$ genes play opposite roles in rice bacteria interactions. Plant Physiol. 151:936-948.

Ülker, B. and Somssich, I. E. 2004. WRKY transcription factors: from DNA binding towards biological function. Curr. Opin. Plant Biol. 7:491-498.

Van Verk, M. C., Pappaioannou, D., Neeleman, L., Bol, J. F. and Linthorst, H. J. 2008. A novel WRKY transcription factor is required for induction of PR-1a gene expression by salicylic acid and bacterial elicitors. Plant Physiol. 146:1983-1995.

Wang, H., Hao, J., Chen, X., Hao, Z., Wang, X., Lou, U., Peng, Y. and Guo, Z. 2007. Overexpression of rice WRKY89 enhances ultraviolet B tolerance and disease resistance in rice plants. Plant Mol. Biol. 65:799-815.

Wu, K., Guo, Z., Wang, H. and Li, J. 2005. The WRKY family of transcription factors in rice and Arabidopsis and heir origins. DNA Res. 12:9-26.

Wu, Q., Song, J., Sun, Y., Suo, F., Li, C., Luo, H., Liu, Y., Li, Y., Zhang, X., Yao, H., Li, X., Hu, S. and Sun, C. 2009. Transcription profiles of Panax quinquefolius from flower, leaf and root bring new insights into genes related to ginsenosides biosynthesis and transcriptional regulation. Physiol. Plant 138:13449.

Wu, X., Shiroto, Y., Kishitani, S., Ito, Y. and Toriyama, K. 2009. Enhanced heat and drought tolerance in transgenic rice seedlings overexpressing OsWRKY11 under the control of HSP101 promoter. Plant Cell Rep. 28:21-30.

Xie, Z., Zhang, Z.-L., Zou, X., Huang, J., Ruas, P., Thompson, D. and Shen, Q. J. 2005. Annotations and functional analyses of the rice $W R K Y$ Gene superfamily reveal positive and negative regulators of abscisic acid signaling in aleurone cells. Plant Physiol. 137:176-189.

Xie, Z., Zhang, Z. L., Hanzlik, S., Cook, E., Shen, Q. J., Zhang, Y. J. and Wang, L. J. 2005. The WRKY transcription factor superfamily: its origin in eukaryotes and expansion in plants. BMC Evol. Biol. 5:1-12.

Xie, Z., Zhang, Z. L., Zou, X., Yang, G, Komatsu, S. and Shen, Q. J. 2006. Interactions of two abscisic-acid induced WRKY genes in repressing gibberellins signaling in aleurone cells. Plant J. 46:231-242.

Zhang, Y. J. and Wang, L. J. 2005. The WRKY transcription factor superfamily: its origin in eukaryotes and expansion in plants. BMC Evol. Biol. 5:1-12.

Zhang, J., Cao, M. L., Huang, Y. B. and Wu, B. J. 2007. Study of $h r p N(C S D S 001)$ and the gene expression profile of Arabidopsis thaliana induced by Harpin (CSDS001). Yi Chuan 29:62936.

Zhang, J., Peng, Y. and Guo, Z. 2008. Constitutive expression of pathogen inducible $O s$ WRKY31 enhances disease resistance and affects root growth and auxin response in transgenic rice plants. Cell Res. 18:508-521.

Zhang, Z. L., Shin, M., Zou, X., Huang, J., Ho, T. H. D. and Shen, Q. J. 2009. A negative regulator encoded by a rice $W R K Y$ gene represses both abscisic acid and gibberellins signaling in aleurone cells. Plant Mol. Biol. 70:139-151. 